\title{
Estratégia de capacitação em cirurgia palpebral baseada em modelo animal
}

\author{
Animalmodel-based strategy of palpebralsurgery training
}

\author{
João França Lopes ${ }^{1}$ \\ Flávio Buzalaf ${ }^{2}$ \\ Beatriz Yae Hanaoka ${ }^{3}$ \\ Suzana Matayoshi ${ }^{4}$ \\ Henrique Shiguekiyo Kikuta ${ }^{4}$
}

\section{RESUMO}

Objetivo: Desenvolvimento de método de treinamento prático em cirurgia palpebral baseado em modelo animal, orientado para o oftalmologista geral e para o iniciante em cirurgia palpebral. Métodos: $O$ modelo porcino foi selecionado devido à semelhança com relação à pálpebra humana. Após breve estudo dirigido com material didático abordando aspectos básicos em cirurgia palpebral e roteiro prático detalhado dos procedimentos a serem realizados, passou-se ao treinamento prático. As técnicas de cirurgia palpebral abordadas foram ressecção pentagonal com reconstrução direta e por planos da pálpebra e técnica original de Bick. O treinamento foi monitorizado cuidadosamente por instrutores com ampla experiência no assunto. Cada aluno tinha à disposição 4 pálpebras. Resultados: Quatorze oftalmologistas foram treinados por meio deste modelo no $1^{\circ} \mathrm{Curso}$ de Cirurgia Palpebral em Animal em dezembro de 2001. Ao final do treinamento, os alunos se mostraram mais confiantes na realização dos procedimentos realizados, acreditando que a experiência havia contribuído positivamente para o desenvolvimento prático em cirurgia palpebral. Conclusão: Omodelo animal porcino de treinamento pode ser etapa valiosa na transição da esfera teórica para a habilitação prática em Plástica Ocular, e pode ser utilizado como estratégia eficaz na capacitação em ressecção pentagonal com reconstrução direta e por planos da pálpebra e técnica original de Bick.

Descritores: Pálpebras/cirurgia; Modelos animais; Capacitação; Cirurgia/educação; Procedimentos cirúrgicos operatórios/educação

\section{INTRODUĈ̃̃O}

Em oftalmologia, existem patologias cujo tratamento envolve o conhecimento de técnicas cirúrgicas de correção palpebral. Dentro do universo variado das afecções palpebrais, existem duas técnicas cirúrgicas básicas que, selecionadas por sua grande versatilidade, podem ter várias indicações. A ressecção pentagonal com reconstrução direta e por planos da pálpebra e a técnica original de $\mathrm{Bick}^{(1)}$ constituem modalidades terapêuticas de ampla aplicabilidade clínica ${ }^{(2)}$, sendo de grande auxílio na correção de alterações palpebrais como traumas ${ }^{(3)}$, tumores, entrópio(4), ectrópio, flacidez palpebral e paralisia facial com flacidez de pálpebra inferior. $\mathrm{O}$ domínio destas duas técnicas permite ampliar a capacidade de resolução para uma grande parte das afecções palpebrais presentes no cotidiano do oftalmologista.

Na programação de congressos e simpósios oftalmológicos é habitual o espaço para laboratórios de suturas e de facoemulsificação. Estes já estão estabelecidos como modalidades eficazes de aprendizado cirúrgico. Na área 
de plástica ocular, infelizmente poucas são as oportunidades de treinamento laboratorial. Propõe-se aqui um modelo de capacitação em cirurgia palpebral baseado em modelo animal, orientado para o oftalmologista geral e para o iniciante em cirurgia palpebral. Este relato descreve o programa de treinamento e as técnicas de ressecção pentagonal com reconstrução direta e por planos da pálpebra e técnica original de Bick.

\section{MÉTODOS}

\section{Desenho do estudo}

Este estudo consiste de um programa de treinamento laboratorial para ensino de técnicas básicas de cirurgia palpebral, empregando modelo porcino. O público-alvo do programa foi composto por oftalmologistas gerais. O conteúdo teórico consistiu na apresentação de aspectos básicos de suturas e cicatrização em cirurgia palpebral, etiopatogenia das alterações palpebrais cirúrgicas mais comuns e descrição das técnicas cirúrgicas empregadas, bem como indicações e complicações. Os assistentes acompanharam as explanações por meio de material didático impresso e multimídia.

\section{Seleção do modelo porcino e técnicas de cirurgia palpebral}

Foram utilizados porcos da raça Sus scrofa. Foram obtidas cabeças frescas de animais abatidos segundo normas da Vigilância Sanitária da região metropolitana da cidade de São Paulo. Após higienização e limpeza, os espécimens foram preparados e dispostos em bancadas com campos cirúrgicos com exposição da região palpebral, em laboratório cirúrgico adequado para uso de modelos animais.

As técnicas de cirurgia palpebral abordadas foram ressecção pentagonal com reconstrução direta e por planos da pálpebra e técnica original de Bick (Figura 1). O treinamento foi coordenado por dois médicos assistentes do Setor de Plástica Ocular da Clínica Oftalmológica do HCFMUSP e equipe de oito monitores com ampla experiência no assunto. Os alunos foram organizados em duplas, e cada par tinha à disposição dois espécimens. Enquanto um dos alunos realizava os procedimentos em um dos olhos, o seu par auxiliava e instrumentava, sob orientação direta de um dos monitores. Os coordenadores circulavam pelas bancadas respondendo dúvidas.

\section{RESULTADOS}

O modelo porcino (Sus scrofa) foi considerado o melhor modelo didático para aplicação no presente curso, devido ao fato de ter pálpebras bastante semelhantes à pálpebra humana e apresentar tamanho adequado das estruturas para o manuseio cirúrgico, e também pela disponibilidade de obtenção.

Quatorze oftalmologistas foram treinados através deste programa, aplicado no $4^{\circ}$ Congresso de Oftalmologia da USP e $3^{\text {o }}$ Congresso de Auxiliar de Oftalmologia da USP, em dezembro de 2001. Todos os alunos executaram as cirurgias propostas (ressecção pentagonal com reconstrução direta e por planos da pálpebra e técnica original de Bick.) em 4 pálpebras animais. Os monitores e coordenadores puderam discutir e demonstrar as particularidades de cada procedimento. Todos os passos cirúrgicos foram revistos pelos monitores, e os deslizes técnicos prontamente apontados e corrigidos. Ao final do treinamento, foi realizada uma sessão aberta de discussão sobre o modelo aplicado, sendo que os alunos se mostraram mais confiantes na realização dos procedimentos realizados, acreditando que a experiência havia sido positiva para maior capacitação prática em cirurgia palpebral.

\section{DISCUSS ÃO}

Devido à grande frequiência das alterações palpebrais, é importante para o oftalmologista geral e para o iniciante em plástica ocular a familiarização com as técnicas de correção cirúrgica que se destinam ao tratamento de tais patologias. Das inúmeras estratégias de intervenção, duas podem ser selecionadas pela ampla aplicabilidade em diversas situações clínicas e relativa facilidade de execução: ressecção pentagonal com reconstrução direta e por planos da pálpebra e técnica original de Bick. Em Plástica Ocular, assim como em outras subespecialidades iminentemente cirúrgicas, a capacitação prática é fundamental. O modelo animal de treinamento constitui uma valiosa etapa de transição da esfera teórica para a habilitação prática em Plástica Ocular, oferecendo subsídios importantes para a realização final das cirurgias em seres humanos. Constitui-se atualmente relevante forma de ensino em diversas áreas ${ }^{(5)}$, apesar de ser pouco utilizada.

A pálpebra porcina apresenta, assim como a humana, 2 lamelas distintas (Figura 2). Da pele para a conjuntiva, apresenta as seguintes camadas: epitélio escamoso, tecido conjuntivo denso, tecido conjuntivo frouxo (semelhante ao tarso, com estruturas glandulares) e epitélio conjuntival.

Apesar das semelhanças anatômicas oculares entre porcos e seres humanos, como tamanho e presença de estruturas básicas correspondentes ${ }^{(6-7)}$, diversos fatores relevantes não são comparáveis, incluindo espessura da pele palpebral e posição dos olhos. Apesar destas diferenças, o porco permanece como animal de escolha para diversos estudos em plástica ocular ${ }^{(6,8)}$. Este modelo não inclui avaliação pós-operatória de modelos vivos, o que poderia revelar mais precisamente os resultados de cada cirurgia realizada pelo aluno em treinamento. Entretanto, as considerações possíveis indicam que a abordagem é satisfatória.

O modelo porcino para treinamento em cirurgia palpebral se mostrou adequado e bastante promissor. Terminado o laboratório, os alunos mostraram maior segurança para a realização das técnicas ensinadas. Um modelo de avaliação pré e póstreinamento está em formulação para melhor caracterização dos resultados desta estratégia de ensino, que em nossa experiência mostrou-se bastante válida. 


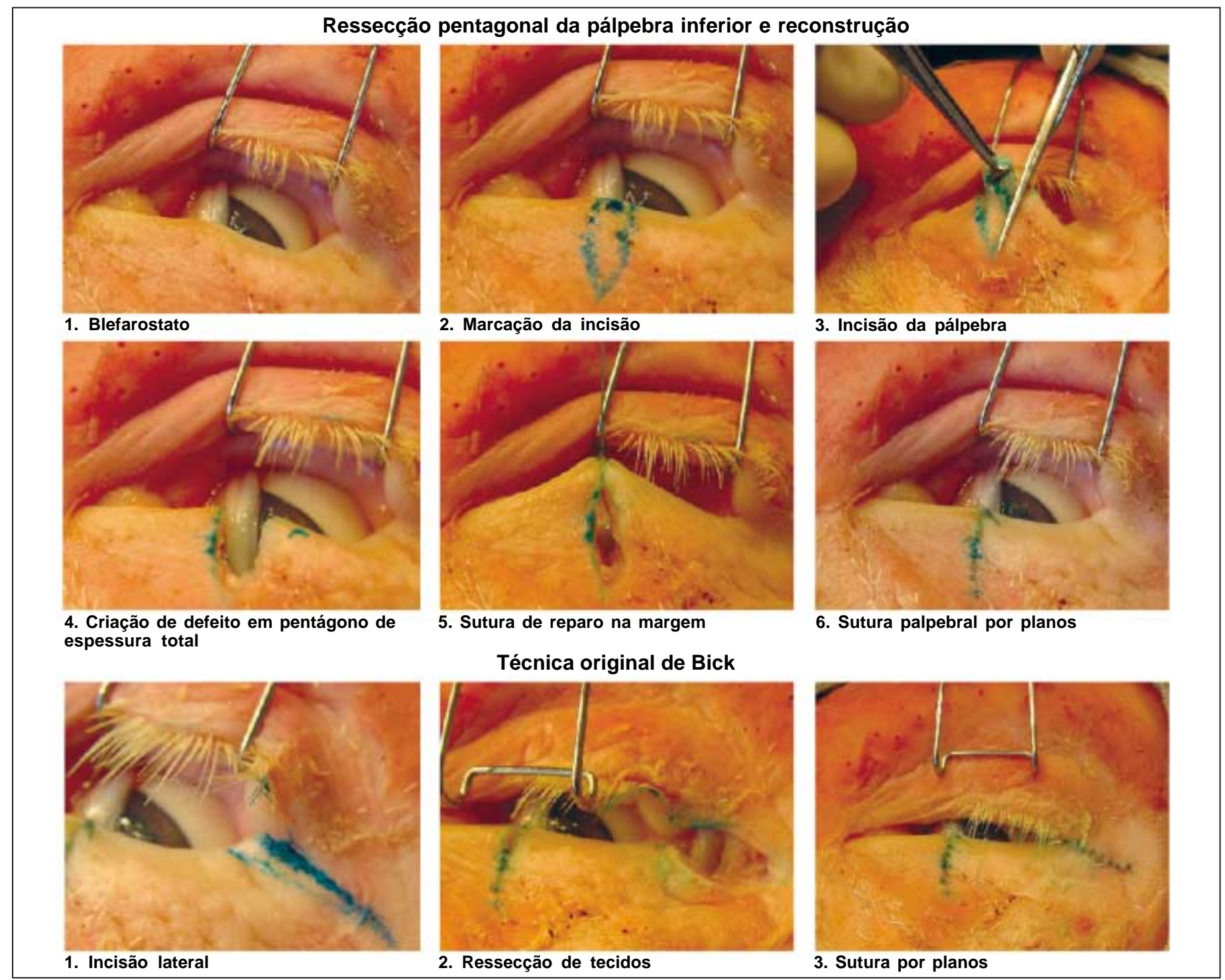

Figura 1

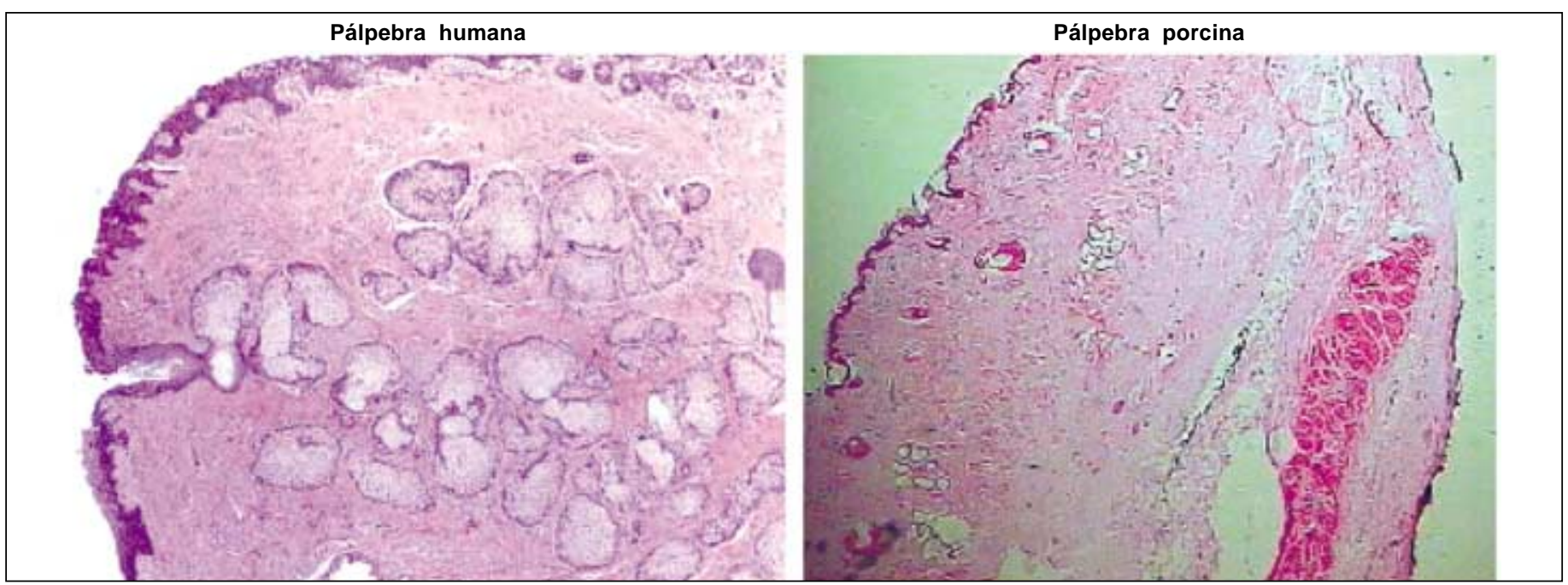

Figura 2 


\section{ABSTRACT}

Objective: Development of an animal model for practical instruction of eyelid surgery, directed to the general ophthalmologist and to the beginner in ophthalmic plastic surgery. Methods: The porcine model was selected due to its similarity with the human lid anatomy. After a quick guided study with instructive material concerning basic aspects in eyelid surgery, with detailed instructions on the procedures to be performed, the practical training was started. The trained surgery techniques were pentagonal resection with direct reconstruction of the eyelid and Bick's original technique. The training was carefully monitored by instructors with wide experience in the subject. Each student used 4 lids. Results: 14 ophthalmologists were trained using this model, applied in

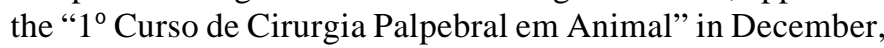
2001. At the end of the model, the students showed a high degree of subjective skill confidence, believing that the experience had contributed positively to their practical development in eyelid surgery. Conclusion: The porcine animal model can be an important step in the transition of the theoretical sphere to practical skill qualification in ocular plastic surgery, and it can be used as an effective instruction strategy for pentagonal resection with direct reconstruction of the eyelid and Bick's original technique.

Keywords: Eyelids/surgery; Models, animal; Training; Surgery/ education; Surgical procedures, operative/education

\section{REFERÊNCIAS}

1. Bick MW. Surgical management of orbital tarsal disparity. Arch Ophthalmo 1966;75:386-9.

2. Caldato R, Soares E. Entrópio. In: Soares EJC, Moura EM, Gonçalves JOR. Cirurgia plástica ocular. São Paulo: Roca; 1997. p.171-84.

3. Kikuta HS, Couto Jr. AS. Traumas palpebrais. In: Moreira Jr. C, Freitas D, Kikuta H. S. Trauma ocular. Rio de Janeiro:Cultura Médica; 1997. p.143-60.

4. Danks JJ, Rose GE. Involutional lower lid entropion: to shorten or not to shorten?. Ophthalmology 1998:105:2065-7.

5. Janick L, DeNovo RC, Henry RW. Plastinated canine gastrointestinal tracts used to facilitate teaching of endoscopic technique and anatomy. Acta Anat (Basel) 1997;158:48-53

6. Suner S, Simmons W, Savitt DL. A porcine model for instruction of lateral canthotomy. Acad Emerg Med 2000;7:837-8.

7. Diesem CD. Cap. 47, Sense Organs and common integument. In: Getty R, editor. Sisson and Grossman's the anatomy of domestic animals. $5^{\text {th }}$ ed. Philadelphia: Saunders; 1975. p.1410-2.

8. Fay AM, Pieroth L, Rubin PA. An animal model of lower eyelid spacer grafting with acellular dermis. Ophthal Plast Reconstr Surg 2001;17:270-5.

\section{ABO ELETRÔNICO}

\section{A versão eletrônica dos Arquivos Brasileiros de Oftalmologia com tex tos completos está disponível em:}

- ABO -

A rquivos Brasileiros de 0 ftalmologia

http:/ / www.abonet.com.br

- Scielo - Scientific Electronic Library 0 nline

http:/ / www.scielo.org

- Free Medical J ournals - http:/ w ww. freemedicaljournals.com 